\title{
Idoso portador de diabetes mellitus: relevância epidemiológica para a atenção em Odontologia
}

Elderly bearer of mellitus diabetes: epidemiologic relevance for attention in

Odontology

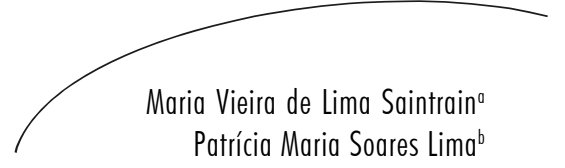

\section{Resumo}

Objetivos: conhecer a prevalência do diabetes mellitus, doenças auto-referidas e saúde bucal em idosos residentes na Comunidade do Dendê. Métodos: Pesquisa quantitativa, exploratória, documental, sob aprovação do COÉTICA. Levantaram-se dados pelo Núcleo de Atenção Médica Integrada-NAMI da população atendida em visitas domiciliares pela Universidade de Fortaleza nos anos de 2000 a 2005. De 8.463 indivíduos visitados, $546(6,5 \%)$ eram idosos, sendo $96(17,6 \%)$ portadores de diabetes mellitus. Destes, 63 idosos, 41 mulheres e 22 homens (idade média de 69,8 anos $\pm 6,9 \mathrm{DP})$, participaram da segunda pesquisa, quando se realizou exame bucal e aplicação de um questionário. Computaram-se os dados pelo software

Palavras-chave: Idoso. Diabetes mellitus. Saúde bucal. Análise quantitativa. Comunidade do Dendê, Fortaleza CE "Statistical Package for Social Science" - SPSS versão 10. Resultados: das doenças auto-referidas, destacam-se: hipertensão [52(82,5\%)], distúrbio da visão [50(79,4\%)], audição $[20(31,7 \%)]$, problemas osteoarticulares $[10(15,9 \%)]$, distúrbio da fala $[9(14,3 \%)]$ e colesterol alto $[6(9,5 \%)]$. Quanto à saúde bucal: - desdentados [30(47,6\%)], lesões da mucosa bucal [22(34,9\%)], maloclusão [11(17,5\%)] 59 pacientes buscaram atendimento clínico; 46 usavam medicamentos; 43 seguiam dieta alimentar; 57 possuíam escova dental. Conclusões: a elevada prevalência do diabetes mellitus entre os idosos do Dendê corrobora dados da literatura científica. Necessita-se controle da sua relação com doenças sistêmicas e da cavidade bucal.

\footnotetext{
Universidade de Fortaleza Curso de Odontologia

Pós-Graduação em Saúde Coletiva

Fortaleza, CE, Brasil

Correspondência / Correspondence

Maria Vieira de Lima Saintrain

Rua Irmã Simas, 100/201

60165-220 - Fortaleza-CE, Brasil

E-mail: Mariavieira@bol.com.br
} 
Abstract

Objectives: to know the prevalence of mellitus diabetes, of self-reported diseases and the oral health in elderly from the Dende's Community. Methods: a quantitative, exploratory, documental research, approved by COETICA. All data were collected by the Núcleo de Atenção Médica Integrada - NAMI amongthe population attended at home by the University of Fortaleza from 2000 to 2005. Of 8,463 visited people, $546(6.5 \%)$ were elderly, $96(17.6 \%)$ bearer of mellitus diabetes. Among the 63 elderly, 41 women and 22 men (average age 69.8 years old +_ 6.9 DP) participated

Keywords: Aged. Diabetes mellitus. Oral health. Quantitative analysis. Comunidade do Dendê, Fortaleza City CE in the second research. Oral exams and a questionnaire were performed and analyzed through the software Statistical Package for Social Science - SPSS version 10. Results: The self-reported diseases were: hypertension [52(82.5\%)], vision disturbs $[50(79,4 \%)]$, hearing disturbs $[20(31,7 \%)]$, osteoarticular problems $[10(15,9 \%)]$, speech disturbs $[9(14,3 \%)]$ and high cholesterol [6(9,5\%)]. Considering the oral health - toothless [30(47,6\%)], lesion in the oral mucosal $[22(34,9 \%)]$, bad occlusion $[11(17,5 \%)]$. Among 63 elderly, 59 searched the clinic services at NAMI, 46 used medicines; 43 followed diets; 57 had toothbrushes. Conclusion: The high prevalence of mellitus diabetes among the elderly from Dende confirms the scientific literature data. There is a need to control its relation with systemic diseasse and oral cavities.

\section{INTRODUÇÃO}

Em razão da melhoria da qualidade de vida, do avanço tecnológico e da ciência ocorridos na área da saúde, a população mundial está envelhecendo e, mais cedo do que se espera, os cirurgiõesdentistas atenderão a um grande número de pacientes idosos. Portanto, na Odontologia, em relação aos idosos, os profissionais devem acrescentar conhecimento sobre as modificações psíquicas, somáticas e comportamentais que comprometem o paciente geriátrico, para poderem exercer um papel de importância e obter êxito na sua atuação.

Dentre as patologias que ensejam preocupação no atendimento à população idosa, o diabetes mellitus requer dos cirurgiões-dentistas cuidados especiais.

$\mathrm{Na}$ lição de Barcellos et al., ${ }^{1}$ o diabetes mellitus caracteriza-se por hiperglicemia crônica com distúrbios no metabolismo de carboidratos, gordura e proteína, como conseqüência de defeitos da secreção de insulina, ação da insulina ou em ambos. O diagnóstico é dado quando os sintomas estão presentes, como poliúria, polidipsia, polifagia, emagrecimento e, nos casos mais graves, desidratação, acidose e distúrbio no nível de consciência, associado a glicosúria.

Lauda et al. ${ }^{2}$ classificam de diabetes tipo I o insulinodependente. Esse tipo é próprio do organismo que produz substâncias que destroem progressivamente as células do pâncreas. Os indivíduos acometidos têm tendência a cetoacidose e costumam ser pessoas magras. O tipo II é o não-insulinodependente e refere-se às pessoas que não tendem a cetoacidose, podendo ser tratados com hipoglicemiantes orais e/ou dietas. 
O diabético tipo II geralmente é obeso e a insulina é produzida pelas células do pâncreas com deficiência e/ou causada pela resistência dos tecidos periféricos à insulina. ${ }^{2,3}$

De acordo com os indicadores do Sistema Único de Saúde - SUS, ${ }^{4}$ atualmente, no Brasil, o diabetes mellitus está entre as dez causas líderes de morte, atingindo o percentual de $4,38 \%$ dos óbitos da população. As mortes precoces relacionadas a essa enfermidade poderiam ser evitadas se os diabéticos e seus componentes familiares tivessem conhecimentos satisfatórios sobre a doença, visando ao tratamento adequado e à conseqüente prevenção das complicações, melhorando sua qualidade de vida. ${ }^{5}$

No que se refere à Odontologia, Elias ${ }^{6}$ considera a inflamação gengival, hiperplasia gengival, bolsas periodontais ativas, abscessos periodontais recorrentes, candidíase oral, perda óssea progressiva e cicatrização lenta do tecido periodontal como sinais e sintomas clínicos periodontais de importância para os cuidados no tratamento odontológico dos pacientes idosos diabéticos.

No entendimento de Boock, ${ }^{7}$ as pesquisas não visam a prolongar a vida indefinidamente, mas reconhecer os mecanismos do processo de envelhecimento, contribuindo para a prevenção de enfermidades no idoso, e fazer parte do alicerce sobre o qual se desenvolverão estratégias para prover serviços de saúde. Sendo a saúde bucal estreitamente relacionada à saúde geral, o autor considera motivo para que o cirurgião-dentista não fique alheio aos programas, sejam em que nível for, que visem a melhorias para a saúde dos idosos. Consoante Lauda et al., ${ }^{2}$ há uma escassa programação de educação e de capacitação profissional em diabetes, assim como uma falta de recursos no controle e na prevenção da moléstia.

Nesta pesquisa, visa-se a contribuir para a informação e o reconhecimento do quadro epidemiológico vivenciado pelos idosos diabéticos, podendo assim oferecer orientações em Educação em Saúde. Pretende-se que as informações e orientações a este respeito possam contribuir para a formação de uma política de saúde que oriente o paciente diabético e, na perspectiva de Elias, ${ }^{6}$ forme equipes multidisciplinares no envolvimento de um diagnóstico e, por conseguinte, na conduta e tratamento mais apropriado ao paciente.

Esta pesquisa teve como objetivo conhecer a prevalência do diabetes mellitus, a situação epidemiológica baseada nas doenças auto-referidas e a saúde bucal em pacientes idosos de 60 anos e mais, residentes em uma comunidade de baixa renda localizada no Município de Fortaleza, Estado do Ceará, Brasil.

O conhecimento sobre a prevalência do diabetes mellitus, sua relação com as doenças autoreferidas e a saúde bucal em idosos podem contribuir significativamente a seus portadores, mediante informações e orientações em Educação em Saúde, sobretudo no que se refere aos agravos na cavidade bucal. Aos portadores desta afecção, torna-se importante despertar o interesse e o conhecimento de sua sintomatologia e referido tratamento. Espera-se contribuir para a informação e o re- 
conhecimento do quadro epidemiológico, oferecendo aos idosos portadores do diabetes mellitus orientações sobre a doença e estimular o autocuidado em saúde.

\section{METODOLOGIA}

Tratou-se de estudo de prevalência, pesquisa de natureza quantitativa, exploratória e documental, realizada no Município de Fortaleza-Ceará, especificamente na Comunidade do Dendê. A população-alvo foi constituída por idosos de 60 anos e mais.

A Comunidade do Dendê possui aproximadamente 15.000 habitantes, que têm como referência para a atenção à saúde o Núcleo de Atenção Médica Integrada - NAMI, pertencente à Universidade de Fortaleza - UNIFOR. O NAMI oferece ao SUS ${ }^{4}$ atendimento em atenção básica e secundária pelas diferentes especialidades, tais como Medicina, Odontologia, Enfermagem, Fisioterapia, Terapia Ocupacional e Fonoaudiologia.

O estudo teve início em 2005, por meio de uma pesquisa documental junto ao NAMI. Nela foram levantados os dados secundários e informatizados da população atendida em visitas domiciliares pelos professores e alunos do Centro de Ciências da Saúde da Universidade de Fortaleza, de 2000 a 2004. Nesse período, a população visitada compreendeu um total de 8.463 indivíduos. Destes, 546 $(6,5 \%)$ eram de idosos de 60 anos e mais, entre os quais foram identificados $96(17,6 \%)$ como portadores do diabetes mellitus.
Com a finalidade de traçar o perfil de saúde e da situação socioeconômica (idade, sexo, renda e escolaridade) foram identificados os pacientes cuja história indicou a presença desta patologia. A Comunidade do Dendê é dividida em microáreas designadas por cores, obedecendo à seguinte distribuição dos idosos identificados: vermelha 16 (25\%), azul 15 (23\%), verde $11(17,5 \%)$, laranja $6(9,5 \%)$, amarela $5(7,9 \%)$, lilás 5 $(7,9 \%)$, e salmão 5 (7,9\%).

Considerou-se como critério de seleção e inclusão da amostra: idoso de 60 anos e mais de ambos os sexos, portadores de diabetes mellitus, residentes na Comunidade do Dendê e cadastrados no NAMI.

No segundo momento, após identificação da amostra, realizou-se a pesquisa de campo. Foi utilizado como instrumento de pesquisa um questionário elaborado com suporte na ficha de cadastro da família, segundo o modelo do Ministério da Saúde e adotada pelo NAMI. O instrumento contém variáveis de identificação do paciente, doenças auto-referidas e informações básicas médicas e sociais. Ao questionário foi acrescida uma ficha contendo variáveis específicas para o levantamento de saúde bucal, tais como: atividade de cárie, sangramento gengival, cálculo dentário, maloclusão, lesões na mucosa bucal, uso e necessidade de prótese, e higiene bucal.

Dos 96 idosos identificados como diabéticos, 33 foram excluídos da pesquisa, pois no momento da visita e revisita (quando necessária) para a coleta de dados, 16 haviam muda- 
do da comunidade, nove faleceram e oito se recusaram a participar. Portanto, a pesquisa foi desenvolvida com um total de 63 idosos, que receberam visita domiciliar para a aplicação do questionário e realização do exame bucal.

O exame bucal foi realizado por único examinador, no domicílio de cada paciente, sob iluminação natural, de forma visual, utilizando espelho bucal, sonda periodontal e gases, devidamente esterilizados. Na verificação de sangramento gengival, utilizou-se da sonda periodontal OMS n ${ }^{\circ} 621$ Trinity, de fabricação nacional. Esta sonda apresenta uma esfera de $0,5 \mathrm{~mm}$ de diâmetro na sua ponta ativa, própria para uso do Índice Periodontal Comunitário - IPC. Foram examinados dez dentes indicadores dos sextantes $(17,16,11,26$, 27, 37, 36, 31, 46, 47); contudo, nos pacientes sem dentes indicadores, examinaram-se todos os dentes remanescentes. A sonda foi inserida com o mínimo de pressão e percorrida suavemente pelo sulco gengival, tendo sido codificado em 0 para ausência de sangramento e 1 quando da presença do mesmo.

Previamente ao início da fase experimental, a pesquisadora realizou calibração direta intra-examinador. Para a padronização e sistematização das informações, o questionário e o exame bucal foram realizados em cinco pacientes idosos da referida comunidade, sendo que o exame bucal foi refeito junto aos mesmos pacientes após 15 dias do primeiro exame, não havendo discordância nos resultados. Foram realizadas modificações no questionário para melhor adequá-lo à compreensão do entrevistado.
Os dados foram analisados estatisticamente, por intermédio do software Statistical Package for Social Science - SPSS versão 10, sendo do tipo descritiva a análise estatística.

Foram condições essenciais, no protocolo da pesquisa, a autorização do fiel depositário dos materiais arquivados, o termo de consentimento livre e esclarecido dos idosos participantes da pesquisa e o cumprimento dos preceitos éticos em pesquisa conforme a Resolução no 196/96, do Conselho Nacional de Saúde, ${ }^{8}$ tendo sido aprovado pelo Comitê de Ética em Pesquisa - COÉTICA, da UNIFOR, expressa dita aprovação no Parecer n ${ }^{\circ} .506 / 2004$.

\section{RESULTADOS}

Dentre as características dos 63 idosos pesquisados, ressalta-se que a idade variou de $60 \mathrm{a}$ 89 anos (média 69,8 anos \pm 6,9 desvio-padrão), constituída por 41 mulheres $(65,1 \%)$ e 22 homens (39,9\%). Predominou uma população de baixa renda, na qual cinco idosos (7,9\%) tinham renda familiar mensal abaixo de 100,00 reais; $41(65,1 \%)$ entre 100,00 a 300,00 reais; dez $(15,9 \%)$ entre 300,00 a 600,00 reais e apenas um ganhava mais de 1.000,00 reais. A maioria dos idosos era aposentada [48 (76,2\%)]. Quanto à escolaridade, $42(66,7 \%)$ eram alfabetizados e $21(33,3 \%)$ não sabiam ler nem escrever.

Na tabela 1, observa-se a prevalência das enfermidades ou doenças auto-referidas discriminadas pelos pacientes idosos portadores de diabetes mellitus. 
Tabela 1 - Distribuição de freqüência de doenças auto-referidas na amostra. Fortaleza-CE, 2005.

\begin{tabular}{lcc}
\hline Doenças auto-referidas & $\mathrm{N}$ & $\%$ \\
\hline Hipertensão & 52 & 82,5 \\
Distúrbio da visão & 50 & 79,4 \\
Distúrbio da audição & 20 & 31,7 \\
Òsteoarticulares & 10 & 15,9 \\
Distúrbio da fala & 9 & 14,3 \\
Colesterol alto & 6 & 9,5 \\
Gastrite & 2 & 3,2 \\
Alzheimer leve & 2 & 3,2 \\
\hline
\end{tabular}

Fonte: dados da pesquisa

Por meio da tabela 2, é possível iden- população de idosos participantes do tificar a condição de saúde bucal da estudo.

Tabela 2 - Distribuição de freqüência da condição de saúde bucal da amostra. Fortaleza-CE, 2005.

\begin{tabular}{llc}
\hline Condição de saúde bucal & N & $\%$ \\
\hline Desdentado total & 30 & 47,6 \\
Dentados & 33 & 52,4 \\
Lesões da mucosa bucal & 22 & 34,9 \\
Maloclusão & 11 & 17,5 \\
\hline
\end{tabular}

Fonte: dados da pesquisa

Pela tabela 3, visualiza-se a condição de dores de diabetes mellitus que ainda possuíam saúde bucal da população de idosos porta- dentes.

Tabela 3 - Distribuição de freqüência da condição de saúde bucal dos idosos dentados Fortaleza-CE, 2005.

\begin{tabular}{lcc}
\hline Condição de saúde bucal & N & $\%$ \\
\hline Atividade de cárie & 27 & 81,8 \\
Cálculo dentário visível & 21 & 63,6 \\
Sangramento gengival à sondagem & 16 & 48,5 \\
Necessidade de Exodontia & 16 & 48,5 \\
Necessidade de Endodontia & 6 & 18,2 \\
\hline
\end{tabular}

Fonte: dados da pesquisa 
A tabela 4 identifica a preferência dos pacientes na busca de assistência. Nestes dados, os pacientes podem ter se referido a uma ou mais categorias.

Tabela 4 - Distribuição de freqüência da procura de assistência profissional pelos idosos. Fortaleza-CE, 2005.

\begin{tabular}{lcc}
\hline Assistência/profissional & $\mathrm{N}$ & $\%$ \\
\hline Medica & 56 & 88,9 \\
Enfermagem & 16 & 25,4 \\
Fisioterapeuta & 12 & 19,0 \\
Odontológica & 9 & 14,3 \\
Nutricionista & 5 & 7,9 \\
Psicológica & 2 & 3,2 \\
\hline
\end{tabular}

Fonte: dados da pesquisa

No total de 63 idosos portadores de diabetes, 59 pacientes buscaram atendimento clínico no Núcleo de Atenção Médica Integrada; 46 faziam uso de medicamentos; 43 seguiam dieta alimentar; 57 possuíam escova dental e seis não usavam escova dental para a higiene bucal.

\section{DISCUSSÃO}

No rastreamento aleatório referente aos anos de 2000 a 2004, de idosos residentes na Comunidade do Dendê, observou-se um percentual de $17,6 \%$ desta mesma população portadores de diabetes mellitus. No final da década de 1980, estimou-se que no Brasil o diabetes ocorria em 3\% a 17\% entre as faixas de 30-39 e de 60-69 anos. ${ }^{9}$

Considerando os 63 idosos do estudo, obteve-se maior prevalência de diabetes mellitus para os idosos do sexo feminino [41 (65,1\%)], em relação ao masculino [22 (39,9\%)], dados compatíveis com os obtidos na literatura. ${ }^{10}$
Embora neste estudo se tenha utilizado o critério de definição do diagnóstico de diabetes mellitus de forma auto-referida e não através de confirmação dos médicos, verifica-se que a pesquisa revelou dados coerentes com outras investigações. A prevalência encontrada no presente estudo $(17,6 \%)$ mostrou-se aproximada aos resultados dos estudos de Souza et al., ${ }^{11}$ cujo percentual detectado foi de $16,3 \%$ e $14,3 \%$ de idosos diabéticos na população pesquisada por Pacce et al. ${ }^{10}$ Dias da Costa et al. ${ }^{12}$ dentre diferentes critérios de diagnóstico do diabetes, obtiveram $10,3 \%$ de prevalência para o diagnóstico auto-referido e confirmado por médico e/ou glicemia >126mg.

A exemplo de Goldenberg et al. ${ }^{13}$ considera-se o diagnóstico auto-referido como tendo importante papel na detecção da doença, fator coadjuvante da busca ativa, evidenciando o perfil da população, enquanto aponta para a diversidade de ações a serem direcionadas na implementação da prevenção da doença, levando em conta a realidade social concreta. 
O alto índice de analfabetismo (33,3\%) observado para os idosos da Comunidade do Dendê mostra-se semelhante à realidade brasileira obtida pela Pesquisa Nacional de Amostra por Domicílio de 1998 - PNAD, na qual foi detectado o percentual de $39,2 \%{ }^{14} \mathrm{~A}$ falta de escolaridade pode interferir no dia-adia do idoso, ocasionando dificuldades ao manusear os medicamentos, seguir dietas ou prescrições, com riscos de ocorrer troca de medicação, trazendo prejuízos à sua saúde, sendo necessária orientação redobrada dos profissionais para seus acompanhantes ou cuidadores, na prevenção de possíveis enganos. Esta real condição justifica a lição de que a verdadeira expressão da pobreza do idoso no Brasil está relacionada às situações de baixo salário e escolaridade. ${ }^{15}$

Quanto aos problemas de saúde apresentados por esta população, e que na literatura se destacam como fatores associados a diabetes mellitus, cita-se a hipertensão arterial, na ordem de $82,5 \%$, superior aos dados encontrados por Souza et al. ${ }^{11}$ cujo percentual de $50 \%$ também visualiza a alta prevalência desta enfermidade junto aos idosos. O controle da hipertensão é de fundamental importância, haja vista que tem implicação no risco cardiovascular duas vezes mais do que nos hipertensos não-diabéticos, ainda que possa contribuir para acelerar lesões da retina, no plano renal e acidente vascular cerebral..$^{16}$ As doenças isquêmicas cardiovasculares são mais freqüentes e mais precoces em indivíduos com diabetes, comparativamente aos demais. ${ }^{4}$

Verificou-se que o distúrbio da visão se mostrou responsável pelo elevado percentual de $79,4 \%$. Pinto \& Moretto, ${ }^{17}$ com o objetivo de analisar características de idosos diabéticos, identificaram nesses pacientes o percentual de 24,28\% de doenças oftalmológicas, e um total de 15,14\% apresentando retinopatia diabética.

Os dados são corroborados pelo Ministério da Saúde, segundo o qual o diabetes mellitus desenvolve complicações crônicas, classificadas como microvasculares (retinopatia, nefropatia e neuropatia) e macrovasculares (doença arterial coronariana, doença cerebrovascular e vascular periférica), responsáveis por expressiva morbimortalidade, com taxas de mortalidade cardiovascular e renal, cegueira, amputação de membros e perda de função e qualidade de vida muito superior à de indivíduos sem diabetes. ${ }^{4}$

Neste estudo, a perda auditiva apresentouse como problema de freqüência elevada $(31,7 \%)$. Maia \& Campos $^{18}$ concluíram que, à luz dos conhecimentos atuais, há indícios de que o diabetes mellitus possa causar perda auditiva, mas não se pode afirmar que exista correlação nítida de causa e efeito. Nos estudos de Marchiori \& Gibrin, ${ }^{19}$ a acuidade auditiva em $97,2 \%$ dos pacientes com diabetes mellitus mostrou-se neurossensorial com rebaixamento nas agudas. As autoras concluíram haver associação direta entre diabetes mellitus e perdas auditivas, principalmente nas freqüências agudas.

Um total de 46 idosos $(73,0 \%)$ fazia uso de medicamentos, o que corrobora os achados de Piccini et al. ${ }^{20}$ consoante os quais, no Nordeste brasileiro, a maioria dos idosos, com 
um total de $85 \%$, usa medicamentos. Da mesma forma, a consulta médica foi identificada com o mesmo perfil de utilização entre os idosos do Nordeste (89\%) e da população de idosos diabéticos do Dendê (88.9\%). Por se tratar, no entanto, de idosos portadores de diabetes mellitus, seria de se esperar que o uso de medicamentos e a utilização do serviço médico atingissem sua totalidade. Esta última possivelmente se complementa pelas consultas de enfermagem. Pela fala dos pesquisados, a atuação multidisciplinar em saúde é mais valorizada nos últimos anos, embora se tenha percebido que os idosos tiveram atendimento com poucos tipos de profissionais, não havendo atendimento integral.

Com relação aos hábitos alimentares, 43 idosos referiram seguir dieta alimentar, mas apenas cinco declararam procurar orientação do nutricionista. Neste contexto, o Ministério da Saúde ${ }^{9}$ recomenda a terapia nutricional como parte fundamental do plano terapêutico do diabetes. É importante salientar que o NAMI, em parceria com o SUS, constitui Unidade de Referência para esta população e dispõe do especialista em Nutrição na sua equipe multidisciplinar.

Os idosos pesquisados apresentaram sua saúde bucal bastante comprometida, não diferindo de estudos recentes. ${ }^{21}$ Além do edentulismo, na monta de $47,6 \%$, a prevalência de lesões da mucosa bucal $(34,9 \%)$ apresentouse diretamente relacionada aos idosos portadores de algum tipo de prótese dentária, haja vista que os 22 casos de lesões foram identificados nestes pacientes.
A difícil cicatrização, inerente ao paciente diabético, associada à má higiene bucal e uso de próteses parciais mal adaptadas, certamente, contribuem para o sangramento da gengiva percebido em $25,4 \%$ dos pesquisados, evidenciando, com maior freqüência, os agravos relacionados a problemas periodontais.

Estes sinais e sintomas clínicos periodontais, quando em pacientes idosos diabéticos, constituem dificuldades e maiores necessidades de cuidados e atuação de tratamento odontológico com equipe multiprofissional e interdisciplinar.

O tratamento periodontal, quando precedido da administração sistêmica de antibióticos, melhora o controle glicêmico metabólico do paciente, porquanto a presença de infecções em diabéticos aumenta a resistência à insulina, agravando a condição da doença e complicando o seu controle metabólico., ${ }^{4,22}$ Ressalta-se a importância do conhecimento do cirurgião-dentista ante as alterações sistêmicas, assim como o diálogo entre Odontologia e Medicina, para que o paciente seja visto como um todo e não parte dele, elevando assim os índices de sucesso terapêutico na diferentes profissões.

Com relação ao sangramento gengival e ao acúmulo de tártaro, como em qualquer tipo de infecção, estes problemas periodontais podem dificultar o controle do diabetes. A presença de infecção leva à estimulação da resposta inflamatória, resultando em situação de estresse, que aumenta a resistência dos tecidos à insulina, dificultando o controle da doença. ${ }^{11}$ 


\section{CONCLUSÃO}

De acordo com os dados observados, pode-se concluir pela existência da elevada prevalência de diabetes melittus entre os idosos da Comunidade do Dendê. Evidenciaramse, nesses pacientes, complicações crônicas em especial a hipertensão arterial, distúrbios da visão e da audição, e grande número de lesões da mucosa bucal associadas à má condição de saúde bucal.

Ressalta-se a necessidade de maior informação e mais controle do diabetes mellitus em relação às doenças sistêmicas e da cavidade bucal, ligadas diretamente às complicações metabólicas que dificultam de forma bidimensional a prevenção e a cura.

\section{NOTAS}

Cirurgiã-dentista, Professora Adjunto do Mestrado em Saúde Coletiva e Curso de Odontologia da Universidade de Fortaleza - UNIFOR.

b Cirurgiã-dentista, Curso de Odontologia da Universidade de Fortaleza - UNIFOR. E-mail: patricinhaslima@hotmail.com

\section{AGRADECIMENTOS}

Agradecemos ao Conselho Nacional de Desenvolvimento Científico e Tecnológico (CNPq) pelo apoio financeiro em forma de bolsa de iniciação científica.

\section{REFERÊNCIAS}

1. Barcellos IF, Halfon VLC. Oliveira LF, Barcellos Filho I. Conduta Odontológica em paciente diabético. RBO 200057 (6):407-410.

2. Lauda PA, Silveira BL, Guimarães MB. Manejo Odontológico do paciente diabético. JBC. Jornal Brasileiro de Odontologia Clínica 1998; 2 (9): 81-87.

3. Melgaço CA. Diabetes Mellitus e a doença periodontal: revisão da literatura. JBE 2002; 3 (9):100-104.

4. Brasil. Ministério da Saúde. Organização PanAmericana da Saúde. Painel de indicadores do SUS. Brasília: Ministério da Saúde; 2006 61p.

5. Duran ECM, Cocco MIM. Software educativo sobre diabetes mellitus para profissionais de saúde: etapas de elaboração e desenvolvimento. Rev Latino-am Enfermagem 2003 11(1): 104-7.

6. Elias R. Diabetes Mellitus. In: Odontologia de alto risco - Pacientes Especiais. Ed. REVINTER. RJ-RJ. 1995. Cap. 8, p. 95-115.

7. Boock ECP. Odontologia geriátrica. Odonto 1991; I (3): 88-90.

8. MS. Ministério da Saúde. Conselho Nacional de Saúde. Resolução Nº196 de 10 de outubro de 1996. Brasília: Decreto de Delegação de Competência de 12 de novembro de 1991.

9. MS (Ministério da Saúde). Diabetes Mellitus. Cadernos de Atenção Básica - n. ${ }^{\circ} 16$ Brasília DF; 2006. 56p.

10. Pacce AE, Ochoa-Vigo K, CaliriMH, Fernandes APM. O conhecimento do Diabetes Mellitus no processo de autocuidado. Rev Latino-am Enfermagem 2006; 14(5) On-line: www.eerp.usp.br/rlae

11. Souza LM, Morais EP, Barth QCM. Características demográficas, socioeconômicas 
e situação de saúde de idosos de um programa de saúde da família de porto alegre, Brasil. Rev Latino-am Enfermagem 2006 14(6) On-line: www.eerp.usp.br/rlae.

12. Dias da Costa JS, Olinto MTA, Assunção MCF, Gigante DP, Macedo S, Menezes AMB. Prevalência de Diabetes Mellitus em Pelotas, RS: um estudo de base populacional.Revista de Saúde Pública 2006; 40(3):542-5.

13. Goldenberg P, Schenkman S, Franco LJ. Prevalência de diabetes mellitus: diferenças de gênero e igualdade entre os sexos. Rev. Bras. Epidemiol. 2003; 6 (1):18-28.

14. IBGE. Pesquisa Nacional por Amostra de Domić́lios 1998; Rio de Janeiro: 2001.

15. Romero, DA. Diferenciais de gênero no impacto do arranjo familiar no status de saúde dos idosos brasileiros. Ciência \& Saúde Coletiva. 7(4):777-94. São Paulo: 2002.

16. Alvarenga C. Hipertensão arterial na Diabete Melittus tipo 2- evidência para a abordagem terapêutica. Rev Port Clin Geral 2005; 21:597604.
17. Pinto AB, Moretto MB. Diabetes Mellitus e Fatores de Risco em Pacientes Ambulatoriais. NewsLab 2004; edição 66 p-106-118.

18. Maia CAS, Campos CAH. Diabetes Mellitus como causa de perda auditiva. Rev Bras Otorrinolaringol. 2005; 71(2): 208-14.

19. Marchiori LLM, Gibrin PCD. Diabetes Mellitus: Prevalência de Alterações Auditivas. Arq Brás Endocrinol Metab 2003; 47(1):8286).

20. Piccini RX, Facchini LA, Tomasi E, Thumé E, Silveira DS, Siqueira FV, Rodrigues MA. Necessidades de saúde comuns aos idosos: efetividade na oferta e utilização em atenção básica à saúde. Ciência e Saúde Coletiva 2006; 11(3):657-667.

21. Monti LM; Justi MM, Farjado RS, Zavanelli AC. Análise comparada da saúde bucal do idoso na cidade de Araçatuba Rev. Bras. Geriatr. Gerontol. 2006; 9 (2).

22. Brondani MA, Brondani AR, Bós AJG. Diabetes e Periodontite - A hora e a vez da medicina periodontall. JBM.Jornal Brasileiro de Medicina 2002; 82(1/2): 12-14. 
1 